\title{
Anomalous scaling for Lagrangian velocity structure functions in fully developed turbulence
}

\author{
Guo-Wei He* \\ Laboratory for Nonlinear Mechanics, Institute of Mechanics, Chinese Academy of Sciences, Beijing 100190, China
}

(Received 17 May 2009; revised manuscript received 21 July 2010; published 3 February 2011)

\begin{abstract}
A hierarchical structure model is developed for anomalous scaling of the Lagrangian velocity structure functions in fully developed turbulence. This model is an extension of the Eulerian hierarchical structure model of She and Leveque [Phys. Rev. Lett. 72, 366 (1994)] to the Lagrangian velocity structure functions, where the straining and sweeping hypotheses are used to build up the relationship between the singular scalings of Lagrangian and Eulerian intermittent structures. The Lagrangian scaling exponents obtained from the straining hypothesis are in good agreement with the experimental results of the Bodenschatz group.
\end{abstract}

DOI: 10.1103/PhysRevE.83.025301

PACS number(s): 47.27.E-, 05.40.-a, 47.27.Jv

The Kolmogorov 1941 theory [1] (hereafter referred to as K41) proposes that universal scaling behaviors exist for smallscale fluctuations in fully developed turbulence. The K41 theory can be presented in either the Eulerian or Lagrangian frames. Previous work [2] focused on the scaling exponents of the Eulerian velocity structure functions (VSFs). Only recently has the interest grown in the scaling exponents of Lagrangian VSFs [3] due to two groups' experiments [4,5]. The experiments found that strong intermittency exists in the Lagrangian VSFs [6], which leads to the large deviations of the Lagrangian scaling exponents from the K41 theory $[7,8]$. The deviations call for a theoretical description. An exact expression for Lagrangian scaling exponents deduced from the Navier-Stokes equations is still unavailable. Hence, it is necessary to develop a phenomenological model for the correction to Kolmogorov's prediction based on the physics of the Navier-Stokes equations. In this Rapid Communication, we will develop such a simple model to analytically predict the Lagrangian scaling exponents.

Since both Lagrangian and Eulerian statistical quantities are determined by the same turbulent flows, there must exist a relationship between these quantities. In the multifractal formulism, a multifractal dimension spectrum uniquely determines the scaling exponents and vice versa. Borgas [9] formulates a relationship between the Eulerian and Lagrangian multifractal dimension spectra. The relationship can be used to calculate the Lagrangian multifractal dimension spectra from the Eulerian ones and yield the Lagrangian scaling exponents. The Lagrangian scaling exponents thus obtained are in good agreement with the experimental measurements for lower orders but less good agreement with them for high orders [7]. Recently, Beck [10] has developed a superstatistical model for Lagrangian scaling exponents, and Zybin et al. [11] have calculated the Lagrangian scaling exponents using the vortex filaments. Using the multifractal formalism, the She and Leveque (SL) model [12] introduces a Eulerian hierarchical structure to describe the Eulerian scaling exponents with its successes. The present work introduces a Lagrangian hierarchy structure to develop a simple model for the Lagrangian scaling exponents without any adjustable parameters.

\footnotetext{
*Author to whom all correspondence should be addressed: hgw@lnm.imech.ac.cn or guoweihe@yahoo.com
}

We consider the Lagrangian VSFs of positive integer order $p$ :

$$
S_{p}(\tau)=\left\langle\left[v_{i}(t+\tau)-v_{i}(t)\right]^{p}\right\rangle \equiv\left\langle\left(\delta_{\tau} v\right)^{p}\right\rangle,
$$

where $v_{i}(i=x, y, z)$ are the velocity components along a single particle path and the repeated indices imply a summation. The ensemble averages are defined as the summation of all samples of particle trajectories. Due to the stationarity and homogeneity in fully developed turbulence, the Lagrangian VSFs are only dependent on time increment $\tau$. The K41 theory implies that the Lagrangian VSFs are solely determined by the average energy dissipation rate $\epsilon$ and the time increment $\tau$ in the inertial subrange. A dimensional analysis yields the normal scaling $S_{p}(\tau) \sim \tau^{\zeta_{p}}$ with $\zeta_{p}=p / 2$. Recent experiments [4,8] and direct numerical simulations (DNSs) [6,7] found that there strong intermittency exists in Lagrangian statistics; that is to say, Lagrangian statistics is largely deviated from Gaussian statistics. The fourth-order local scaling exponents obtained from the experiments and DNS collapse into one curve with a plateau of the slope smaller than 2 [6], while the K41 theory predicts $\zeta_{4}=2$. Xu et al.'s experiment [5] found that the Lagrangian scaling exponents at high orders are deviated from the K41 prediction; that is, $\zeta_{p} \neq p / 2$.

A number of phenomenological models have been proposed to address the intermittency. Among them, the SL model is particularly powerful for predicting the Eulerian scaling exponents. The SL model is based on the following three hypotheses [12,13].

(1) The first is the Eulerian version of the Kolmogorov refined similarity hypothesis (KRSH). Kolmogorov's 1962 refined similarity hypothesis (K62) introduces a locally averaged energy dissipation rate $\epsilon_{\ell}$ to account for intermittency of small-scale fluctuations, that is, $\left\langle\left(\delta_{\ell} u\right)^{p}\right\rangle \sim\left\langle\epsilon_{\ell}^{p / 3}\right\rangle \ell^{p / 3}$, where $\delta_{\ell} u$ is the Eulerian velocity difference across a distance $\ell$.

(2) In the hierarchical structure hypothesis, for each $\epsilon_{\ell}$, the $p$ th-order normalized moment ratio, $\left[\left\langle\epsilon_{\ell}^{p+1}\right\rangle /\left\langle\epsilon_{\ell}^{p}\right\rangle\right] /\left\langle\epsilon_{\ell}^{\infty}\right\rangle$, is related to the next order ratio by

$$
\frac{\left\langle\epsilon_{\ell}^{p+1}\right\rangle}{\left\langle\epsilon_{\ell}^{p}\right\rangle\left\langle\epsilon_{\ell}^{\infty}\right\rangle}=A_{p}^{E}\left[\frac{\left\langle\epsilon_{\ell}^{p}\right\rangle}{\left\langle\epsilon_{\ell}^{p-1}\right\rangle\left\langle\epsilon_{\ell}^{\infty}\right\rangle}\right]^{\beta^{E}}
$$

where $\left\langle\epsilon_{\ell}^{\infty}\right\rangle=\lim _{p \rightarrow \infty}\left[\left\langle\epsilon_{\ell}^{p+1}\right\rangle /\left\langle\epsilon_{\ell}^{p}\right\rangle\right]$ describes the most intermittent structures, $\beta^{E}$ is a symmetry parameter independent 
of $p$, and $A_{p}^{E}$ is a constant independent of $\ell$. The superscript $E$ denotes the symbols for the Eulerian quantities.

(3) For the most intermittent structure hypothesis, the limit of the moment ratio can be scaled as $\left\langle\epsilon_{\ell}^{\infty}\right\rangle \sim \ell^{-h^{E}}$, where $h^{E}=$ $2 / 3$ is the singular scaling for the most intermittent structures.

These hypotheses on energy dissipation rates propose an interesting picture for fully developed turbulence: small-scale fluctuations exhibit a scale-invariance so that their moment ratios satisfy the hierarchy structures with two ends: the mean field $\left\langle\epsilon_{\ell}\right\rangle$ and the most intermittent structures $\left\langle\epsilon_{\ell}^{\infty}\right\rangle$. The moment ratio specifies the geometric difference between two successive moments and is further normalized by the limit of moment ratios. This normalization leads to a geometric series, given by

$$
\ln \left[\frac{\left\langle\epsilon_{\ell}^{p+1}\right\rangle}{\left\langle\epsilon_{\ell}^{p}\right\rangle\left\langle\epsilon_{\ell}^{\infty}\right\rangle}\right]=\beta^{E} \ln \left[\frac{\left\langle\epsilon_{\ell}^{p}\right\rangle}{\left\langle\epsilon_{\ell}^{p-1}\right\rangle\left\langle\epsilon_{\ell}^{\infty}\right\rangle}\right] .
$$

The geometric relation (3) suggests the same scaling relation as Eq. (2) does, although it ignores the constant $A_{p}^{E}$. This picture is supported by the energy cascade process and supposed to be held for Lagrangian statistics. We propose the Lagrangian version of these hypotheses as follows.

(1) For the Lagrangian version of $\mathrm{KRSH}, S_{p}(\tau) \sim$ $\left\langle\epsilon_{\tau}^{p / 2}\right\rangle \tau^{p / 2}$, where $\epsilon_{\tau}(t)$ is a local temporal average of energy dissipation rate defined by $\epsilon_{\tau}(t)=\frac{1}{\tau} \int_{t-\tau / 2}^{t+\tau / 2} \epsilon(s) d s$. If we denote $\left\langle\epsilon_{\tau}^{p}\right\rangle \sim \tau^{z_{p}}$, then $\zeta_{p}=p / 2+z_{p / 2}$.

(2) For the hierarchical structure hypothesis, for each $\epsilon_{\tau}$, the $p$ th-order normalized moment ratio, $\left\langle\epsilon_{\tau}^{p+1}\right\rangle /\left[\left\langle\epsilon_{\tau}^{p}\right\rangle\left\langle\epsilon_{\tau}^{\infty}\right\rangle\right]$, is related to the next order ratio by

$$
\ln \left[\frac{\left\langle\epsilon_{\tau}^{p+1}\right\rangle}{\left\langle\epsilon_{\tau}^{p}\right\rangle\left\langle\epsilon_{\tau}^{\infty}\right\rangle}\right]=\beta \ln \left[\frac{\left\langle\epsilon_{\tau}^{p}\right\rangle}{\left\langle\epsilon_{\tau}^{p-1}\right\rangle\left\langle\epsilon_{\tau}^{\infty}\right\rangle}\right],
$$

where $\left\langle\epsilon_{\tau}^{\infty}\right\rangle=\lim _{p \rightarrow \infty}\left[\left\langle\epsilon_{\tau}^{p+1}\right\rangle /\left\langle\epsilon_{\tau}^{p}\right\rangle\right]$ is the limit of moment ratios and $\beta$ is a symmetry parameter independent of $p$.

(3) The singular scaling for the limit of moment ratios is $\left\langle\epsilon_{\tau}^{\infty}\right\rangle \propto \tau^{-h}$, where $\left\langle\epsilon_{\tau}^{\infty}\right\rangle \sim \tau^{-h}$, where $h$ is constant.

The physical picture behind KRSH is that turbulent kinetic energy is transferred from large-scale eddies to small-scale ones in a cascade process. The Lagrangian KRSH describes its temporal aspect, while the Eulerian KRSH describes its spatial aspect. Both of them are determined by a unique dynamics of the energy cascade process. Therefore, the Eulerian statistics can be transformed to the Lagrangian statistics. The transformation can be obtained using dimensional analysis: $\delta_{\ell} u \sim$ $\delta_{\tau} v, \epsilon_{\ell} \sim \epsilon_{\tau}$, and $\ell \sim \tau \delta_{\tau} v$. Substituting those dimensional relations into Kolmogorov's second universality assumption, $\delta_{\ell} u \sim\left(\epsilon_{\ell} \ell\right)^{1 / 3}$, leads to its Lagrangian version $\delta_{\tau} v \sim\left(\epsilon_{\tau} \tau\right)^{1 / 2}$. The same arguments can be made to obtain the Lagrangian hierarchical structure hypothesis (4) and its singular scaling $\left\langle\epsilon_{\tau}^{\infty}\right\rangle \sim \tau^{-h}$. Recent DNSs have shown strong evidence to support the Lagrangian KRSH [14-16]. Another approach to derive Eq. (4) is to assume that both $\epsilon_{\ell}$ and $\epsilon_{\tau}$ obey a joint log-Poisson distribution, which leads to a joint hierarchical structure. A joint hierarchical structure was proposed for the scalars and velocity dissipation rates, which yields a scaling exponent model for passive scalars with a good prediction of experimental data [17]. The possibility has been explored to replace the dissipation rate in the $\mathrm{KRSH}$ and Eq. (4) by the enstrophy, without significant outputs, since the dissipation and enstrophy have the same asymptotic behaviors at infinite Reynolds numbers [18].

The hierarchical structure implies a scaling relation

$$
z_{p+2}-z_{p+1}+h=\left[z_{p+1}-z_{p}+h\right] \beta
$$

which has a general solution

$$
z_{p}=\alpha \beta^{p}+B p+C
$$

The asymptotic property, $\lim _{p \rightarrow \infty}\left(z_{p+1}-z_{p}\right)=-h$ implies $B=-h$. Introducing the boundary conditions $z_{0}=z_{1}=0$, we obtain the solution

$$
z_{p}=-h p+C\left[1-(1-h / C)^{p}\right]
$$

which is the generation function of a log-Poisson process. In the Eulerian hierarchical structure hypothesis, $C^{E}=2$ is taken since it represents the codimension of the most intermittent structures (filaments) [12]. In the present study, $\left\langle\epsilon_{\tau}^{\infty}\right\rangle$ characterizes the most intermittent structures along Lagrangian trajectories. The isocontours for the moment ratios describe the intermittent structures in turbulent flows, and the isocontours of its limits are therefore referred to as the most intermittent structures. It is the Lagrangian version of the most intermittent structure in the Eulerian frame and characterizes the most intermittent structures along the Lagrangian trajectory. In terms of the ergodic theorem, the ensemble of Lagrangian trajectories fills in the entire space of turbulent flows. On the other hand, Lagrangian intermittent structures are swept, by energetic eddies, from the corresponding Eulerian intermittent structures at the previous time. The sweeping process is much faster than the stretching one. Therefore, the Lagrangian intermittent structures move down with small distortion at the sweeping time scales. This implies that the most intermittent structures in the Lagrangian frame have the same codimension as the ones in the Eulerian frame. That is to say, $C=2$. The most intermittent structures dominate the hierarchical structures such that the symmetry parameter $\beta$ is determined by the singular scaling $h$ and codimension $C$. The probabilistic interpretation of $\beta$ offers another interesting approach to determine those parameters; see reference [19] for details.

There are two plausible alternatives for the singular scaling of the most intermittent structures $\left\langle\epsilon_{\tau}^{\infty}\right\rangle$ : the scalings can be obtained either by nonlocal sweeping or by local straining. The decorrelation process in turbulent flows can be described by velocity time correlation (VTC). A Eulerian VTC is defined as the correlation of Eulerian velocities at two times and two locations, while a Lagrangian VTC is defined as the velocity correlation of two particles at two times. Kraichnan [20] develops the sweeping hypothesis for the Eulerian VTC and the straining hypothesis for the Lagrangian VTC. In terms of the sweeping hypothesis, the small-scale eddies are swept by energy-containing eddies so that the Eulerian VTC are mainly determined by the sweeping velocity ( $\mathrm{rms}$ of velocity fluctuation). Tennekes [21] points out that the sweeping 
hypothesis is the stochastic form of Taylor's frozen-flow hypothesis. The straining hypothesis implies that the smallscale eddies are internally stretched and thus the Lagrangian VTC is determined by enstrophy. The DNS data verify both the sweeping hypothesis [22] and the straining hypothesis [23]. The extension of the sweeping hypothesis to passive scalars is also made and verified [24]. Recently, the time correlation models for turbulent shear flows have also been developed [25-28]. The sweeping hypothesis is challenged by the renormalization group (RNG) theory [29] and the experiments [30,31] on large Reynolds number flows. It was found later in [32] that the RNG results discard the sweeping effect at the outset and do not demonstrate inhibition of sweeping. The experiments [30,31] show some evidence against the statistical independence of the energy-containing range and inertial range eddies. This issue is related to the sweeping hypothesis but is not equivalent to the sweeping hypothesis itself. The statistical correlation may be due to the finite Reynolds number effect and possibly decrease with increasing the Reynolds numbers. However, due to the limitation of currently available Reynolds numbers, the conclusion is difficult to make.

The straining hypothesis implies that the time scale is a local property of small-scale eddies. Therefore, a dimensional argument $\left\langle\epsilon_{\tau}^{\infty}\right\rangle \sim(\delta v)^{2} / \tau \sim \tau^{-1}$ suggests $h=1$. On the other hand, the sweeping hypothesis implies that the time scale is a nonlocal property of the energy-containing eddies [22,25,26]. That is to say, $\ell \sim V \tau$, where $V$ is the rms of fluctuation velocities. Using the result from the SL model, $\left\langle\epsilon_{\ell}^{\infty}\right\rangle \sim \ell^{-2 / 3}$, we obtain $\left\langle\epsilon_{\tau}^{\infty}\right\rangle \sim \tau^{-2 / 3}$ and thus $h=2 / 3$.

If the straining hypothesis is used, then $h=1$. The symmetry parameter is $\beta=1 / 2$. The scaling exponent $z_{p}$ becomes

$$
z_{p}=-p+2\left[1-(1 / 2)^{p}\right] .
$$

Using the Lagrangian $\mathrm{KRSH}$, we obtain the straining model

$$
\zeta_{p}=p / 2+z_{p / 2}=2\left[1-(1 / 2)^{p / 2}\right] .
$$

This model predicts $\zeta_{2}=1$ and thus the Lagrangian frequency spectrum of energy $E_{L}(\omega) \sim \omega^{-2}$. Figure 1 shows the comparisons of the straining model with the experimental data [5]. It can be seen that the straining model is in good agreement with the experimental data: all scaling exponents from the straining model are close to the experimental data within the error bars. The straining model predicts that the Lagrangian scaling exponents increase monotonously with order $p$ and approach 2 as $p \rightarrow \infty$. It implies that the Lagrangian scaling exponents are saturated. The saturation is due to the fact that the linear scaling $p / 2$ is canceled by the singular scaling $-h p$ when $h=1 / 2$.

If the sweeping hypothesis is used, then $h=2 / 3$. The symmetry parameter is $\beta=2 / 3$. The sweeping model is $z_{p}=$ $2 p / 3+2\left[1-(2 / 3)^{p}\right]$, and thus, $\zeta_{p}=p / 6+2\left[1-(2 / 3)^{p / 2}\right]$. It is easily observed from Fig. 1 that the scaling exponents for $p \leqslant 4$ are within the error bars and the exponents for $p>4$ are out of the error bars. For the same parameter $h=2 / 3, \zeta_{p}$ with smaller $C$ can be better matched with the experimental data. However, the model with $h=2 / 3$ does not saturate.

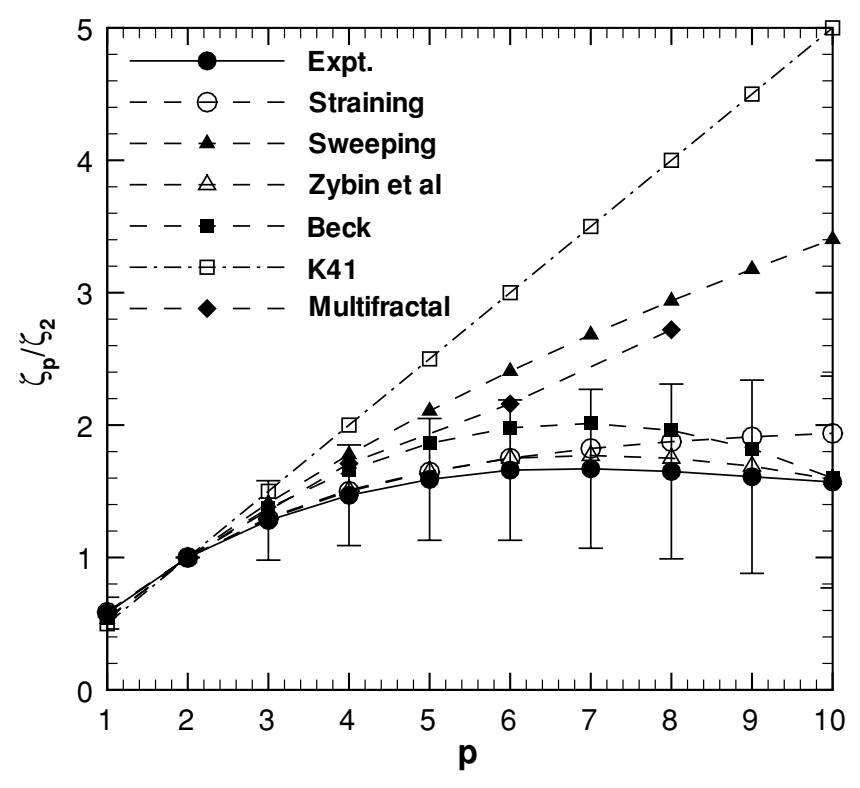

FIG. 1. Scaling exponents $\zeta_{p}$ of the Lagrangian velocity structure functions as a function of order.

The experimental data support the straining model rather than the sweeping model. The straining model indicates that the Lagrangian scaling exponents increase monotonously with its orders and saturate at infinitely large $p$. Zybin et al.'s model [11] predicts that the Lagrangian scaling exponents decrease for $p>7$, and Beck's model [10] predicts that the Lagrangian scaling exponents could decrease to be negative for larger $p$. The decrease of Lagrangian scaling exponents at higher orders need to be further justified, while the decrement observed in the experiments is due to insufficient data samples [5].

We now calculate the multifractal dimensional spectra $D_{s t}$ and $D_{s w}$ for the straining model and the sweeping model, respectively, using the Legendre transform:

$$
\begin{aligned}
D_{s t}(h)= & 1+\frac{2}{\ln (2)}[(\ln (\ln (2))+1) h-h \ln h] \\
D_{s w}(h)= & 1-\frac{2}{\ln (3 / 2)}\left[\left(\ln \left(\ln \frac{3}{2}\right)\right)-1\right]\left(h-\frac{1}{6}\right) \\
& -\frac{2}{\ln (3 / 2)}\left(h-\frac{1}{6}\right) \ln \left(h-\frac{1}{6}\right)
\end{aligned}
$$

Both dimension spectra $D_{s t}(h)$ and $D_{s w}(h)$ are concave with two end points. Their shapes are in agreement with the ones obtained from the experiments $[4,8]$.

Lumley [33] points out that the general problems of relating Lagrangian and Eulerian statistical quantities have been difficult to solve. In some sense, finding such a relationship would imply that one has to solve a particular closure problem. In the multifractal formalism, Borgas [9] develops an approach to calculate the multifractal scaling properties of Lagrangian statistics from the ones of Eulerian statistics. The SL model introduces a hierarchical structure: small-scale fluctuations are related to each other via the most intermittent structures. 
The present work relates the Lagrangian most intermittent structures with the Eulerian ones using either the straining or the sweeping hypothesis. We assume that the Lagrangian VSFs share the same hierarchy structures as the Eulerian ones, but with different singular scalings. The straining hypothesis yields a simple model that is in good agreement with the experimental measurements; the sweeping hypothesis yields another model that does not predict well the scaling exponents from the experiments, especially for higher-order moments. The former correctly describes the stretching of small-scale eddies internally, while the latter introduces the large-scale sweeping into small-scale eddies. We hope that the Lagrangian hierarchical structure model can motivate further understanding of Lagrangian intermittency in turbulence.

This work was supported by the National Natural Science Foundation of China under Project Nos. 10325211, 10628206, and 10732090, the Chinese Academy of Sciences under Innovative Project "Multi-scale modeling and simulation in complex systems" (KJCX-SW-L08), and the National Basic Research Program of China (973 Program) under Project No. 2007CB814800.
[1] U. Frisch, Turbulence: The Legacy of A. M. Kolmogrov (Cambridge University Press, Cambridge, 1995).

[2] K. R. Sreenivasan and R. A. Antonia, Annu. Rev. Fluid Mech. 29, 435 (1997).

[3] F. Toschi and E. Bodenschatz, Annu. Rev. Fluid Mech. 41, 375 (2009).

[4] L. Chevillard, S. G. Roux, E. Leveque, N. Mordant, J.-F. Pinton, and A. Arneodo, Phys. Rev. Lett. 91, 214502 (2003).

[5] H. T. Xu, M. Bourgoin, N. T. Ouellette, and E. Bodenschatz, Phys. Rev. Lett. 96, 024503 (2006).

[6] A. Arneodo, R. Benzi, J. Berg, L. Biferale, E. Bodenschatz et al., Phys. Rev. Lett. 100, 254504 (2008).

[7] L. Biferale, G. Boffetta, A. Celani, B. J. Devenish, A. Lanotte, and F. Toschi, Phys. Rev. Lett. 93, 064502 (2004).

[8] H. T. Xu, N. T. Ouellette, and E. Bodenschatz, Phys. Rev. Lett. 96, 114503 (2006).

[9] M. S. Borgas, Philos. Trans. R. Soc. London, Ser. A 342, 379 (1993).

[10] C. Beck, Phys. Rev. Lett. 98, 064502 (2007).

[11] K. P. Zybin, V. A. Sirota, A. S. Ilyin, and A. V. Gurevich, Phys. Rev. Lett. 100, 174504 (2008).

[12] Z.-S. She and E. Leveque, Phys. Rev. Lett. 72, 336 (1994).

[13] B. Dubrulle, Phys. Rev. Lett. 73, 959 (1994).

[14] R. Benzi, L. Biferale, E. Calzavarini, D. Lohse, and F. Toschi, Phys. Rev. E 80, 066318 (2009).

[15] R. Benzi, L. Biferate, R. Fisher, D. Q. Lamb, and F. Toschi, J. Fluid Mech. 653, 221 (2010).
[16] H. D. Yu and C. Meneveau, Phys. Rev. Lett. 104, 084502 (2010).

[17] G. W. He, S. Y. Chen, and G. Doolen, Phys. Lett. A 246, 135 (1997).

[18] G. W. He, S. Y. Chen, R. H. Kraichnan, R. Y. Zhang, and Y. Zhou, Phys. Rev. Lett. 81, 4636 (1998).

[19] L. Liu and Z.-S. She, Fluid Dyn. Res. 33, 261 (2003).

[20] R. H. Kraichnan, Phys. Fluids 7, 1723 (1964).

[21] H. Tennekes, J. Fluid Mech. 67, 561 (1975).

[22] G. W. He, M. Wang, and S. K. Lele, Phys. Fluids 16, 3859 (2004).

[23] T. Gotoh, R. S. Rogallo, J. R. Herring, and R. H. Kraichnan, Phys. Fluids A 5, 2846 (1993).

[24] P. K. Yeung and B. L. Sawford, J. Fluid Mech. 459, 129 (2002).

[25] G. W. He and J. B. Zhang, Phys. Rev. E 73, 055303(R) (2006).

[26] X. Zhao and G. W. He, Phys. Rev. E 79, 046316 (2009).

[27] G. W. He, G. D. Jin, and X. Zhao, Phys. Rev. E 80, 066313 (2009).

[28] X. Z. He, G. W. He, and P. Tong, Phys. Rev. E 81, 065303 (2010).

[29] V. Yakhot, S. A. Orszag, and Z. S. She, Phys. Fluids A 1, 184 (1989).

[30] A. A. Praskovsky, E. B. Gledzer, M. Y. Karyakin, and Y. Zhou, J. Fluid Mech. 248, 493 (1993).

[31] M. Kholmyansky and A. Tsinober, Phys. Fluids 20, 041704 (2008).

[32] S. Y. Chen and R. H. Kraichnan, Phys. Fluids A 1, 2019 (1989).

[33] J. H. Lumley, in Mecanique de la turbulence (CNRS, Paris, 1962), p. 18 . 